\title{
Precise In-Band OSNR and Spectrum Monitoring Using High-Resolution Swept Coherent Detection
}

\author{
Xiangqing Tian, Yikai Su, Member, IEEE, Weisheng Hu, Lufeng Leng, Member, IEEE, Peigang Hu, Hao He, \\ Yi Dong, Member, IEEE, and Lilin Yi
}

\begin{abstract}
We propose and demonstrate a novel in-band optical signal-to-noise-ratio (OSNR) and spectrum monitoring technique based on swept coherent detection. This technique measures the signal and the in-band amplified spontaneous emission spectra by properly adjusting the polarization states of the signal and the local oscillator. A high resolution of $0.002 \mathrm{~nm}$ is achieved. By integrating the detected spectra of the signal and the noise, the in-band OSNR can be obtained in a very precise manner. We monitor the OSNR values with high accuracy in the range of 10-30 dB.
\end{abstract}

Index Terms-Coherent detection, optical performance monitoring, optical signal-to-noise-ratio (OSNR), spectral analysis.

\section{INTRODUCTION}

$\mathbf{I}^{\mathrm{N}}$ $\mathrm{N}$ FUTURE reconfigurable wavelength-division multiplexed (WDM) networks, each data channel may go through a lightpath that employs multiple wavelength multiplexers/demultiplexers of different types. Although the out-of-band noise can be suppressed by the filtering effects of the multiplexing/demultiplexing components, it is the in-band amplified spontaneous emission (ASE) noise that mainly determines the optical signal-to-noise-ratio (OSNR). Previously reported in-band OSNR monitoring techniques include the orthogonal delayed-homodyne method [1], carrier-to-noise ratio correlation [2], RF spectral dip analysis [3], and polarization-nulling schemes [4], [5]. While OSNR is often used as an indicator for the signal quality, a high-resolution optical spectrum can reveal the fine features of the pulses and provide additional insights into the signal evolution process. Therefore, a technique that can simultaneously and precisely monitor the in-band OSNR, the optical spectrum of the signal, and the filtering effects of cascaded filters would be highly desirable for performance monitoring of optical networks. In this letter, we propose a novel in-band OSNR and spectrum monitoring scheme based on swept coherent detection with polarization control. The signal and the in-band ASE spectra are measured separately by properly adjusting the polarization states of the signal and the wavelength-tunable local oscillator (LO). By

Manuscript received June 27, 2005; revised August 21, 2005. This work was supported in part by the National Natural Science Foundation of China under Grant 60407008/90304002, Shanghai Optical Science and Technology under Grant 04dz05103, and the Shanghai Rising Star Program 04QMX1413.

X. Tian, Y. Su, W. Hu, P. Hu, H. He, and Y. Dong are with State Key Laboratory of Advanced Optical Communication Systems and Networks, Shanghai Jiao Tong University, Shanghai 200030, China (e-mail: yikaisu@ sjtu.edu.cn).

L. Leng is with the New York City College of Technology, City University of New York, NY 11201, USA.

L. Yi is with the Department of Electronic Engineering, Shanghai Jiao Tong University, Shanghai 200240, China.

Digital Object Identifier 10.1109/LPT.2005.859185

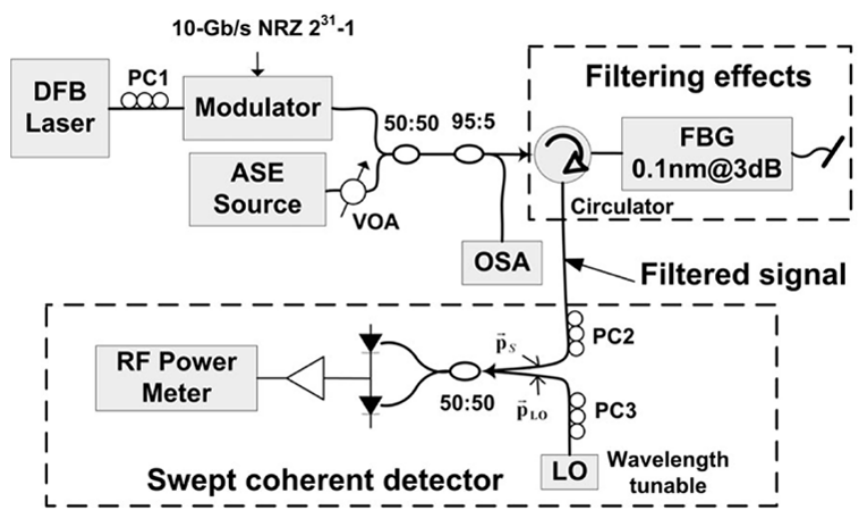

Fig. 1. Schematic for in-band OSNR and spectrum monitoring based on swept coherent detection (PC: polarization controller, FBG: fiber Bragg grating, VOA: variable optical attenuator).

integrating the measured spectra we obtain the in-band OSNR in a very precise manner.

\section{PROPOSED SCHEME}

Fig. 1 shows the proposed schematic to demonstrate the operating principle of in-band OSNR and spectrum monitoring. A distributed-feedback (DFB) laser centered at $1552.52 \mathrm{~nm}$ is externally modulated by a $10-\mathrm{Gb} / \mathrm{s}$ nonreturn-to-zero (NRZ) data sequence with a pattern length of $2^{31}-1$. After the modulator, the optical signal is combined with an ASE source whose power can be adjusted by a variable optical attenuator (VOA) to obtain different OSNR values. 95\% of the combined signal and noise power is launched into a fiber Bragg grating (FBG) filter through a circulator. The FBG filter, with a $3-\mathrm{dB}$ bandwidth of $0.1 \mathrm{~nm}$ centered at $1552.52 \mathrm{~nm}$, is introduced to mimic the filtering effects. The swept coherent detector consists of a wavelength-tunable laser as the LO, a balanced photodetector pair, and a lowbandwidth RF power meter. The balanced photodetector pair is used to eliminate the baseband signal in coherent detection. PC2 and PC3 are used to adjust the polarization states of the signal and the LO, respectively. In practice, a commercially available polarization stabilizer could be employed to maintain and stabilize the polarization states regardless of the input polarizations.

The filtered ASE noise within a narrow bandwidth can be expressed as

$$
E_{\mathrm{ASE}}(t)=\sum_{i}\left|A_{i}\right| \cdot \exp \left[j 2 \pi f_{i} t+j \varphi_{i}(t)\right] \vec{p}_{\mathrm{ASE}, i}
$$

where $A_{i}, \varphi_{i}(t)$, and $\vec{p}_{\mathrm{ASE}, \mathrm{i}}$ are random variables to denote the amplitude, phase, and polarization, respectively, of the ASE 


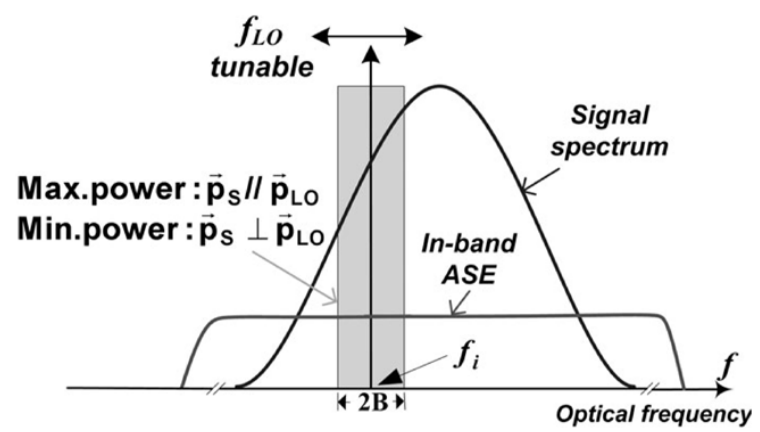

Fig. 2. Operating principle of in-band high-resolution swept coherent detection scheme.

component at frequency $f_{i}$. Similarly, the fields of the signal and the $\mathrm{LO}$ are

$$
\begin{aligned}
E_{S}(t) & =\sum_{k}\left|A_{S, k}\right| \cdot \exp \left[j 2 \pi f_{S, k} t+j \varphi_{S, k}(t)\right] \vec{p}_{S} \\
E_{\mathrm{LO}}(t) & =\left|A_{\mathrm{LO}}\right| \cdot \exp \left[j 2 \pi f_{\mathrm{LO}} t+j \varphi_{\mathrm{LO}}(t)\right] \vec{p}_{\mathrm{LO}}
\end{aligned}
$$

respectively. At the balanced photodetector output, the average electrical power within the bandwidth of the RF power meter can be derived as

$$
\begin{aligned}
\left\langle P_{E}(t)\right\rangle \propto & \left|A_{\mathrm{LO}}\right|^{2} \cdot\left(\sum_{k}\left|A_{S, k}\right| \vec{p}_{\mathrm{LO}} \cdot \vec{p}_{S}\right)^{2} \\
& +\left|A_{\mathrm{LO}}\right|^{2} \cdot\left\langle\left(\sum_{i}\left|A_{i}\right| \vec{p}_{\mathrm{LO}} \cdot \vec{p}_{\mathrm{ASE}, i}\right)^{2}\right\rangle \\
\propto & \left|A_{\mathrm{LO}}\right|^{2} P_{S}\left(\vec{p}_{\mathrm{LO}} \cdot \vec{p}_{S}\right)^{2}+\frac{1}{2}\left|A_{\mathrm{LO}}\right|^{2} P_{\mathrm{ASE}}
\end{aligned}
$$

where $\langle\cdot\rangle$ denotes time average. In (4), we have omitted the signal-ASE beating term because of its low power compared to the LO-ASE term $\left(P_{\mathrm{LO}} \gg P_{S}\right)$. The factor of one-half in the LO-ASE term is due to $\vec{p}_{\mathrm{ASE}, i}$ being a random variable. If the polarization states of the signal and the LO are orthogonal to each other, i.e., $\vec{p}_{s} \perp \vec{p}_{\mathrm{LO}}$, then $\vec{p}_{s} \cdot \vec{p}_{\mathrm{LO}}=0$, and minimal power is detected at the RF power meter as (4) shows. The output is a direct indicator of the in-band ASE power at a specific frequency. When $\vec{p}_{S} / / \vec{p}_{\mathrm{LO}}$, then $\vec{p}_{S} \cdot \vec{p}_{\mathrm{LO}}=1$, and the photodetector pair outputs a maximal RF power. In this case, the signal power and half of the in-band ASE power are detected.

By continuously tuning the wavelength of the LO, the receiver scans over the whole bandwidth of the optical signal and provides a highly resolved optical spectrum. At the RF power meter, only the frequency components that satisfy $\left|f_{\mathrm{LO}}-f_{i}\right|<$ $B$ can be monitored, where $B$ is the bandwidth of the power meter as shown in Fig. 2. In the experiment we simply used an RF spectrum analyzer as the power meter. The detected electrical power at frequency $f_{\mathrm{LO}}$ is linearly related to the optical power at frequency $f_{i}$. In the experiment, to achieve high resolution while keeping a reasonable number of sampling points, we chose the sweeping step of the LO to be $0.002 \mathrm{~nm}$. Therefore, the corresponding bandwidth of the power meter was set to be $130 \mathrm{MHz}$. The measurement time of our scheme can be within minutes with a fast sweeping tunable laser and the corresponding general purpose interface bus (GPIB) interface. The

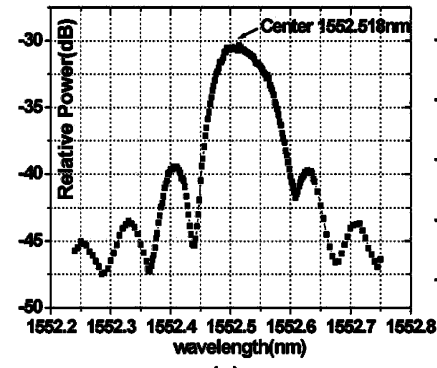

(a)

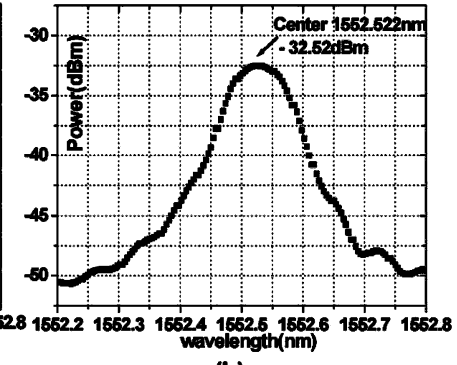

(b)
Fig. 3. In-band ASE spectra measured by (a) swept coherent detection and (b) OSA.

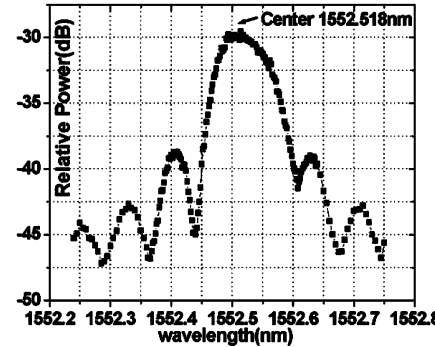

(a) In-band ASE spectrum

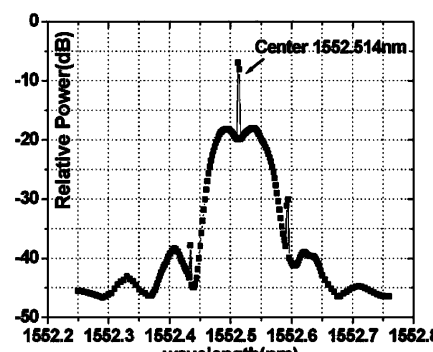

wavelength( $(\mathrm{nm})$

(b) Signal spectrum

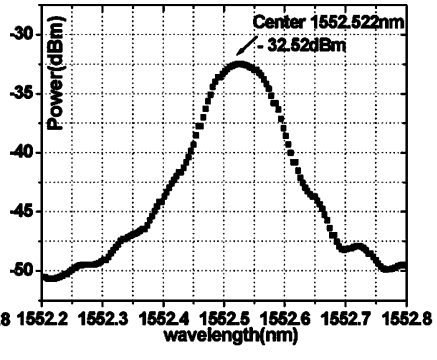

(c) In-band ASE spectrum

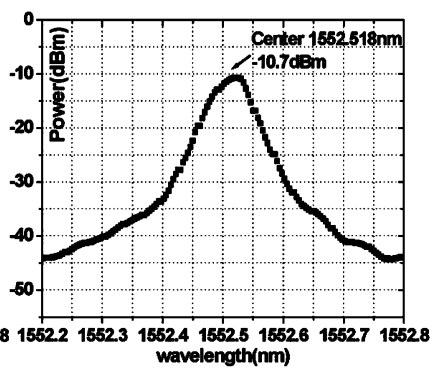

(d) Signal spectrum
Fig. 4. Optical spectra of signal and in-band ASE. (a) and (b) are measured by swept coherent detector, while (c) and (d) are measured by OSA set at $0.07-\mathrm{nm}$ $\mathrm{RB}$

requirement on the power and wavelength stability, the tuning range, the linewidth, and the output power of the tunable local laser can be satisfied by most commercial tunable laser modules.

\section{EXPERIMENTAL RESULTS}

We first measured the in-band ASE by switching off the DFB laser in the transmitter. Here, we assume that the ASE noise has flat power distribution over a wide range of wavelength, so the measured in-band ASE spectrum indicates the filter shape. The result is provided in Fig. 3(a). The sideband peaks and dips of the FBG filter are shown by the in-band ASE. However, with the conventional optical spectrum analyzer (OSA) [Fig. 3(b)], the details of the FBG passband cannot be seen due to insufficient $\mathrm{RB}$ of the OSA $(0.07 \mathrm{~nm})$. We then turn on the DFB laser and measure in-band ASE and signal spectra by properly adjusting the polarization states of the signal and the LO. The results are provided in Fig. 4. We observe that there is little difference between Figs. 3(a) and 4(a), showing that the signal has been properly "blocked" by the orthogonally polarized LO in the detectors, thus only the in-band ASE is detected even when the signal 


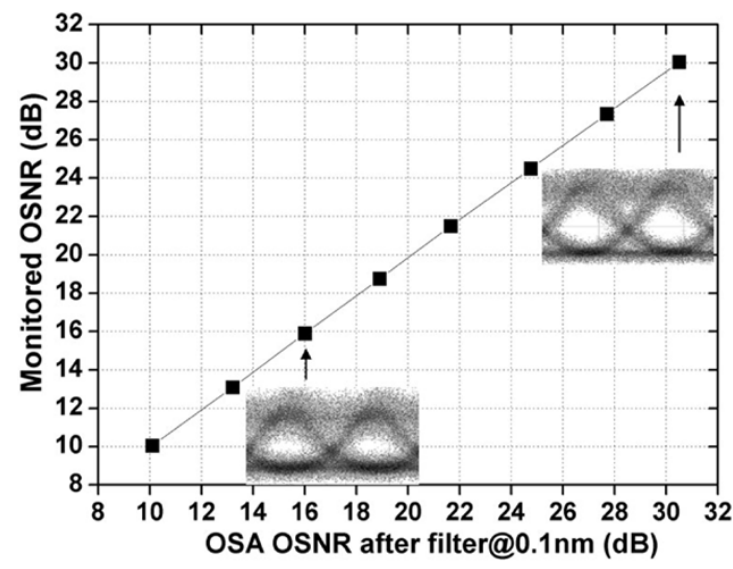

Fig. 5. OSNRs measured by swept coherent detection versus OSNRs measured by the OSA, with all data taken after filter. Insets are eye diagrams of filtered $10-\mathrm{Gb} / \mathrm{s}$ NRZ signal.

is present. In Fig. 4(b), the very fine structures of the NRZ signal are visible, and the sideband tones can be clearly seen using our scheme. The measured results using the OSA are also provided in Fig. 4(c) and (d) for comparison. The in-band ASE spectrum, which reveals the passband shape of the cascaded filters, also can be used to determine the misalignment between the central frequency of the signal and the filters.

It is then straightforward to obtain the in-band OSNR by integrating the measured spectra. Fig. 5 provides the results at different OSNR values. Two eye diagrams for the OSNR values of 16 and $30 \mathrm{~dB}$ are the insets in the figure. The distortion in the eye diagrams is due to tight filtering. Note that after the filter certain spectral components of both the signal and the noise are lost, resulting in different OSNR values before and after the filter. For fair comparisons, the signal and the ASE noise are separately sent into the FBG filter, and the OSA set at $0.1 \mathrm{~nm} \mathrm{RB}$ is used to measure the powers after the filter. The measured OSNR values are used as horizontal-scale data in the figure to compare with the results obtained using the coherent detection scheme. The demonstrated approach, unlike the conventional OSA that requires switching off the signal, enables the measurement of both the signal and the noise spectra. The measured OSNR values using our scheme agree well with the results using the OSA within a maximal difference of $0.5 \mathrm{~dB}$, which occurs at $30-\mathrm{dB}$ OSNR. The discrepancy at high OSNRs can be attributed to incomplete polarization "blocking" in the measurement process, which results in small leakage of the signal and overestimation of the ASE noise power. This is the major limitation on the monitoring range in our scheme. The OSNR monitoring range could be even wider; however, we do not see the necessity to further exploit it since 10-30-dB OSNR is a typical range in practical systems. In the experiment, we did not observe much fluctuation in the monitored OSNR values during the course of the measurement, which shows that reasonable stability can be achieved to enable consistent measurement in our proposed scheme.

It is worth noting that with certain modifications our method can stand the impairment of polarization mode dispersion (PMD). It has been shown that polarization nulling-based monitoring techniques are sensitive to PMD [4]. The state-of-po- larization of the signal varies across the wavelength [6] if the signal is impaired by PMD. This causes signal-power leakage during ASE noise measurement, resulting in spectral distortions and OSNR errors. However, in our scheme, a dynamic polarization controller in the signal lightpath could be employed to measure the maximal and minimal RF powers within a polarization-scanning period. PMD-induced monitoring errors can therefore be eliminated because of the ultranarrow-band measurement process [5].

Recently, it was shown that a high degree of partially polarized noise [7] and interchannel cross-phase modulation (XPM)induced polarization scattering [8] are two sources of errors for the polarization-assisted OSNR monitoring methods. We do not expect large monitoring errors with our scheme in real systems in the presence of polarization dependent loss, especially in systems where the number of spans is less than 30 [7]. On the other hand, our proposed scheme could be susceptible to polarization scattering under certain conditions. However, in high-speed ( $>10 \mathrm{~Gb} / \mathrm{s}$ ) systems, where intrachannel effects are of major concern, or metro/access systems, where interchannel nonlinearity is not dominant, we expect that the monitoring errors are small.

\section{SUMMARY}

We present a simple high-resolution in-band OSNR and optical spectrum monitoring technique based on swept coherent detection. Our scheme eliminates the need for any high-speed components; it is independent of the data rate and particularly attractive for precise OSNR monitoring at lower bit rates due to its ability to achieve fine resolution. OSNR values are measured and compared with the results obtained by the OSA to demonstrate the effectiveness and accuracy of our technique over a wide measurement range of 10-30 dB.

\section{REFERENCES}

[1] C. J. Youn, K. J. Park, J. H. Lee, and Y. C. Chung, "OSNR monitoring technique based on orthogonal delayed-homodyne method," IEEE Photon. Technol. Lett., vol. 14, pp. 1469-1471, Oct. 2002.

[2] G. Rossi, T. E. Dimmick, and D. J. Blumenthal, "Optical performance monitoring in reconfigurable WDM optical networks using subcarrier multiplexing," J. Lightwave Technol., vol. 18, pp. 1639-1648, Dec. 2000.

[3] G. W. Lu, M. H. Cheung, L. K. Chen, and C. K. Chan, "Simultaneous PMD and OSNR monitoring by enhanced RF spectral dip analysis assisted with a local large-DGD element," in Tech. Dig. ECOC'2004, We4.P.092.

[4] J. H. Lee, D. K. Jung, C. H. Kim, and Y. C. Chung, "OSNR monitoring technique using polarization-nulling method," IEEE Photon. Technol. Lett., vol. 13, no. 1, pp. 88-90, Jan. 2001.

[5] M. H. Cheung, L. K. Chen, and C. K. Chan, "PMD-insensitive OSNR monitoring based on polarization-nulling with off-center narrow-band filtering," IEEE Photon. Technol. Lett., vol. 16, no. 11, pp. 2562-2564, Nov. 2004.

[6] G. W. Lu, C. Xie, Y. C. Ku, L. K. Chen, and C. K. Chan, "Enhanced PMD monitoring with frequency-resolved SOP rotation by self phase modulation," IEEE Photon. Technol. Lett, vol. 16, no. 9, pp. 2180-2182, Sep. 2004.

[7] M. D. Feuer, "Measurement of OSNR in the presence of partially polarized ASE,' IEEE Photon. Technol. Lett, vol. 17, pp. 435-437, Jan. 2005

[8] C. Xie and D. C. Kilper, "Influence of polarization scattering on polarization-assisted OSNR monitoring in dense WDM systems with NZ-DSF and Raman amplification," in Tech. Dig. OFC'2005, JWA40. 